\title{
Multi-Tenant Mobile Offloading Systems for Real-Time Computer Vision Applications
}

\author{
Zhou Fang \\ University of California San Diego \\ Mani B. Srivastava \\ University of California Los Angeles
}

\author{
Jeng-Hau Lin \\ University of California San Diego \\ Rajesh K. Gupta \\ University of California San Diego
}

\begin{abstract}
Offloading techniques enable many emerging computer vision applications on mobile platforms by executing compute-intensive tasks on resource-rich servers. Although there have been a significant amount of research efforts devoted in optimizing mobile offloading frameworks, most previous works are evaluated in a single-tenant setting, that is, a server is assigned to a single client. However, in a practical scenario that servers must handle tasks from many clients running diverse applications, contention on shared server resources may degrade application performance. In this work, we study scheduling techniques to improve serving performance in multi-tenant mobile offloading systems, for computer vision algorithms running on CPUs and deep neural networks (DNNs) running on GPUs. For CPU workloads, we present methods to mitigate resource contention and to improve delay using a Plan-Schedule approach. The planning phase predicts future workloads from all clients, estimates contention, and adjusts future task start times to remove or reduce contention. The scheduling phase dispatches arriving offloaded tasks to the server that minimizes contention. For DNN workloads running on GPUs, we propose adaptive batching algorithms using information of batch size, model complexity and system load to achieve the best Quality of Service (QoS), which are measured from accuracy and delay of DNN tasks. We demonstrate the improvement of serving performance using several real-world applications with different server deployments.
\end{abstract}

\section{CCS CONCEPTS}

- Human-centered computing $\rightarrow$ Ubiquitous and mobile computing; • Computer systems organization $\rightarrow$ Cloud computing; Client-server architectures;

ACM Reference Format:

Zhou Fang, Jeng-Hau Lin, Mani B. Srivastava, and Rajesh K. Gupta. 2019. Multi-Tenant Mobile Offloading Systems for Real-Time Computer Vision Applications. In Proceedings of International Conference on Distributed Computing and Networking, Navi mumbai, India, fanuary 4-7, 2019 (ICDCN '19), 10 pages.

https://doi.org/10.1145/3288599.3288634

Permission to make digital or hard copies of all or part of this work for personal or classroom use is granted without fee provided that copies are not made or distributed for profit or commercial advantage and that copies bear this notice and the full citation on the first page. Copyrights for components of this work owned by others than ACM must be honored. Abstracting with credit is permitted. To copy otherwise, or republish, to post on servers or to redistribute to lists, requires prior specific permission and/or a fee. Request permissions from permissions@acm.org.

ICDCN '19, fanuary 4-7, 2019, Navi mumbai, India

(c) 2019 Association for Computing Machinery.

ACM ISBN 978-1-4503-6094-4/19/01 . \$ \$15.00

https://doi.org/10.1145/3288599.3288634

\section{INTRODUCTION}

Workload offloading techniques extend resource-constrained mobile devices with servers that have great computational power. The servers can be either deployed in low-latency and high-bandwidth local clusters that provide timely offloading services, as envisioned by the cloudlet [30], or the cloud that provides best-effort services. Compared to the cloud, the cloudlet has several advantages for latency-sensitive tasks. Sub-second level latency constraint of realtime (RT) mobile tasks can be achieved by low-latency, one-hop, high-bandwidth wireless access to local servers [6, 14]. In addition, it avoids streaming a large amount of data over public networks to the cloud, by aggregating and processing data at the edge servers [36]. Many continuous mobile vision applications that are compute-intensive are made feasible using mobile offloading to the cloudlet. For example, cognitive assistants provide the environmental information to guide vision impaired people [14]. Intelligent personal assistants (IPAs) on smartphones that answer queries in the form of voice and vision information to assist users [17]. As these applications are increasingly popular and ubiquitous on mobile platforms, the computational load on servers raises accordingly driven by this trend.

The server end of a mobile offloading framework is a multi-tenant serving system that executes tasks from independent clients on shared server machines. Scheduling techniques that decide when, where and how long to process each workload are needed to improve the serving performance of offloaded tasks with varying computational overhead, timing and performance requirements. While schedulers in the cloud have been well devised for a wide range of data processing jobs with timing requirements [26, 33], these tasks are on a much larger time scale of makespan and deadline, usually ranging from seconds to hours. They are inadequate for scheduling latency-critical mobile vision queries that require sub-second delays. Moreover, common approaches of maintaining low latency in the cloud that put a low limit on processor utilization are constrained for the cloudlet. The resources owned by a cloudlet are on a much smaller scale, whereas the number of clients may grow uncontrollably. As a result, it may cause a denial of service scenario if the resource utilization must be kept low.

Although schedulers designed specifically for latency-critical offloading systems in the cloudlet are demanding, unfortunately, existing works on mobile offloading usually do not consider the multi-tenant issue, and use a simple single-tenant setting that one client is assigned to one server to evaluate the prototype implementations $[6,14]$.

This paper summarizes our previous works to bridge this gap by designing new scheduling methods to solve the multi-tenant 
problems $[11,12]$. We first present the mobile offloading systems that manage a cluster of servers to execute computer vision algorithms on CPUs [11], as well as deep neural networks (DNNs) on GPUs [12]. Then the details of schedulers are described:

Mitigating Resource Contention: For CPU workloads, we design a system named ATOMS that coordinates future tasks to remove or reduce potential resource contention, and then dispatches incoming tasks to the best server based on the estimated remaining contention on each server. The coordination actions ahead of task start time are based on accurate predictions on future workloads. It uses estimation of wireless network delays and uncertainties, as well as the client-server clock synchronization technique, to get accurate timing information of offloaded tasks.

DNN Adaptive Batching: On GPU servers, we use batching to improve throughputs for DNN queries. We design adaptive batching algorithms considering two Quality of Service (QoS) metrics for query responses: accuracy and delay. The algorithms dynamically select a batch size and a DNN model to process on a GPU in a cluster, with the goal to maximize the serving performance that is an utility function of inference accuracy, delay, and query deadline. We present a simple heuristic algorithm and a more advanced algorithm based on deep reinforcement learning.

The results show that we can effectively reduce workload contention on CPUs using the Plan-Schedule algorithm, using several experiments involving tens of clients and various applications The Plan-Schedule algorithm is able to maintain low end-to-end delays even when the system utilization is very high $(80 \%)$. For adaptive batching of DNN queries, we demonstrate that the QoSaware schedulers improve the serving performance measured by customized response quality functions, by automatically switching to faster DNN models when the query rate is high.

\section{BACKGROUND AND RELATED WORKS}

Mobile Offloading: Recent works on mobile offloading have made substantial progresses in optimizing the system in terms of end-toend delays, power consumption on devices and bandwidth usage of wireless networks. According to how an application is partitioned between local and remote computing resources, offloading frameworks are classified to fine-grained [8,23] and coarsegrained $[6,14,31]$ approaches. Among fine-grained approaches, Wishbone [23] statically partitions an application between the sensor and server based on a data-flow graph of operators. MAUI [8] implements dynamic partitioning based on profiling to achieve energy-aware offloading. Coarse-grained offloading encapsulates an entire application or a processing stage as a computing engine. For example, COSMOS [31] implements a cloud resource manager to improve both offloading performance and monetary cost, which uses VM instances to serve offloaded tasks.

This work focuses on coarse-grained offloading frameworks. Among previous coarse-grained frameworks that provide timely offloading service, Gabriel [14] deploys cognitive engines in a nearby cloudlet that is only one wireless hop away to minimize network latency. Glimpse [6] reduces network delay for object detection in live videos by tracking objects on the client side, using stale results from the server. In this work, we address resource contention that is a fundamental issue for multi-tenant offloading systems, however, not yet emphasized in the previous works.

DNN Serving Systems: DNNs have found widespread use for their versatility and demonstrated value. Despite the excellent performance of DNN models, the high overheads of computation and memory make their deployment on thin client-end devices a challenging task, such as smartphones and wearable devices. Executing DNN inference tasks on cloud-hosted GPU servers provides an attractive alternative that reduces computing delay, as well as power consumption on client-end devices. They have become an important class of mobile workloads that are offloaded to servers. Among mobile offloading systems designed for DNN tasks, MCDNN [15] studied the problem of scheduling variants of DNNs on the mobile and cloud for performance-resource trade-off. Neurosurgeon [19] explored the methods to partition DNN inference between the mobile and server end with the granularity of neural network layers.

It has attracted intense research interests to serve DNN inference tasks in the cloud as a service. A DNN serving system manages trained models and processes queries using the models. The representative systems include the DjiNN project [16] that designs a centralized DNN service infrastructure. It considers DNN models for image, speech and natural language processing (NLP) applications. The system is targeted at warehouse scale servers with high processing throughput as the primary goal. Clipper [7] is a low-latency serving system. It considers delay, throughput and accuracy altogether, and adopts dynamic batching and model selection techniques to improve the service performance. The opensource Tensorflow Serving framework [22] provides a solution for high-performance production-level model serving, with dynamic batching and latency controlling capabilities.

Our work considers real-time DNN inference queries processed in the cloudlet. We present the QoS-aware scheduling algorithms that decide the batch size and model type dynamically for each query to co-optimize delay and accuracy.

\section{MOBILE OFFLOADING SYSTEMS}

We start with the description of a general mobile offloading system, and the computer vision tasks that we use to evaluate the system.

\subsection{System Model}

An offloading request sent by a mobile client $C_{i}$ to the server is denoted as task $T_{j}^{i}$. We ignore the superscript for simplicity when discussing only one client. The life cycle of offloading a task to the server is given in Figure 1. For the task $T_{j}$, it is sent from client at $t_{-}$send $_{j}$, and received by server at $t \_s e r v e r r_{j}$. It is kept in the ready queue until the server has free resource to execute it at $t \_s t a r t_{j}$. It is completed at $t \_$end $d_{j}=t_{-}$start $_{j}+d_{-}$compute $_{j}$, where $d_{-}$compute $_{j}$ is the computing time. The client receives the result back at $t_{-} r e c v_{j}$. For CPU workloads, $T_{j}$ uses $T_{-}$paral $_{j}$ cores in parallel. Because a parallel program may have sequential sections that can not fully utilize all cores, in our model the parallelism $T_{-}$paral $_{j}$ can be a noninteger. The details of obtaining T_paral are given in Section 4.2.

We evaluate a task by E2E delay, which includes the delays of upstream network $d \_u p_{j}$, queueing $d \_q u e u e_{j}$, computing $d \_$compute $e_{j}$ and downstream network $d \_d o w n_{j}$, calculated as:

$$
d_{-} \operatorname{delay}_{j}=t \_r e c v_{j}-t \_s e n d_{j} .
$$




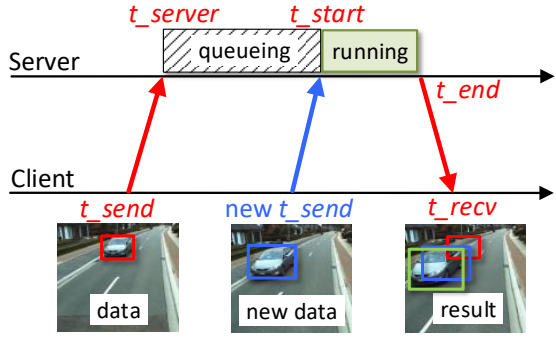

Figure 1: ATOMS predicts processor contention and postpones offloading to avoid queueing delay for tasks running on CPUs.

\subsection{Mobile Vision Applications}

We use a few applications to motivate and evaluate our work: Face Detection: FaceDetect continuously detects human faces from a video stream. We use the Haar feature cascade classifiers [34] implemented in OpenCV [5]. It applies feature filters on small windows. Instead of fully processing all windows, a window is discarded as soon as it fails any stage of filter. The total computing time thus highly depends on the image and may have large variability. Our implementation is single-threaded.

Stereo Feature Matching: FeatureMatch detects interest points in frames from a video stream captured by a binocular camera, extracts sparse features (e.g., SIFT [20], SURF [4]), and matches features. It outputs pairs of matched points. Our implementation computes SURF features of the left and right frames on two threads, then runs brute-force matching on one thread. Because the number of interest points depends on the content of a frame, the computing time can be highly variable.

DNN Object Detection: ObjectDetect detects a set of objects in a video stream. It localizes objects and labels each one with a likeliness score. We use the real-time deep neural network (DNN) based object detection system YOLO [28]. When running on CPUs, the model uses all cores of a CPU in parallel. It has a constant computing time for each video frame. Object detectors are used as GPU workloads as well to evaluate adaptive batching algorithms.

\section{SERVING SYSTEM ON SERVERS}

The offloading framework is composed of a master server and multiple worker servers. The master communicates with clients and dispatches tasks to workers for execution. It is responsible for scheduling tasks. In this section, we present the design of worker component that serves computer vision algorithms as micro-services. A worker hosts one or more computing engines. Each computing engine runs an offloading application encapsulated in a Docker container [1]. A worker receives tasks from the master and execute them on CPUs or GPUs, and processes one task at a time.

\subsection{CPU Workers}

The system for serving tasks running on CPUs is shown in Figure 2. We note the total number of CPU cores of worker $W_{k}$ as $W_{-} c p u_{k}$. A worker can have multiple engines for the same application in order to fully exploit multi-core CPUs. For example, to serve a single thread application on a dual-core machine, two engines should be

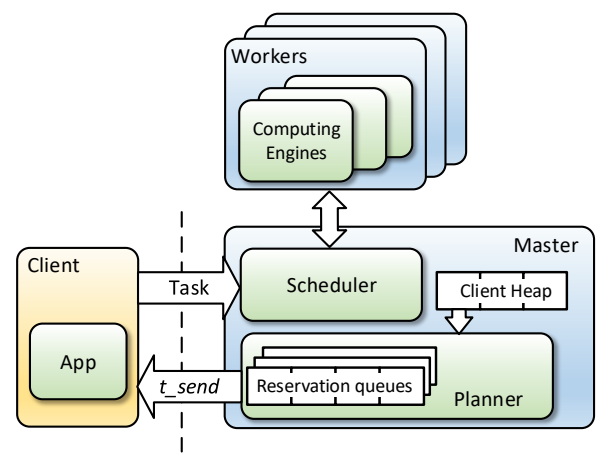

Figure 2: The architecture of the ATOMS system.

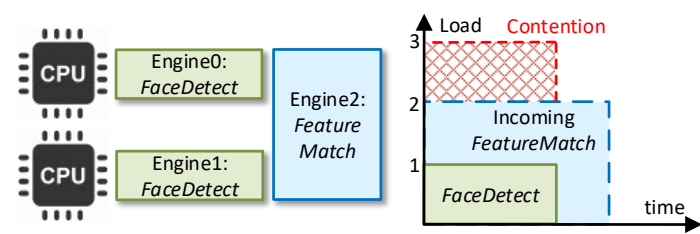

Figure 3: A dual-core worker machine have two engines of FaceDetect $\left(T_{-}\right.$paral $\left.=1\right)$ and one engine of FeatureMatch $\left(T \_\right.$paral $\left.=2\right)$. The plot on right gives an example of processor contention.

hosted. A worker can also host different types of engines to share the machine by multiple applications. In this case, the total workloads of all engines on a worker may exceed the limit of processor resource ( $\left.W_{-} c p u\right)$. Accordingly, we classify workers into two types: reserved worker and shared worker.

On a reserved worker, the sum of processor usage of compute engines never exceeds $W_{-} c p u$. Therefore whenever there is a free computing engine, it is guaranteed that dispatching a task to it does not induce any processor contention. Unlike a reserved worker, the total CPU load on a shared worker may exceed $W_{-} c p u$. See Figure 3, a dual-core machine hosts two FaceDetect engines $\left(T_{-}\right.$paral $\left.=1\right)$ and one FeatureMatch engine $\left(T_{-}\right.$paral $\left.=2\right)$. Both applications are able to fully utilize the dual-core processor. When there is a running FaceDetect task, an incoming FeatureMatch task will cause processor contention. The Load-aware scheduling described in Section 6 is used for shared workers.

\subsection{Measure CPU Computing Time}

Workers measure the computing time of each task (d_compute) and returns it to the master along with the computation result. The measurements are used to predict d_compute for future tasks (Section 6.3).

A straightforward method is measuring the start and end timestamps of a task, and calculating the difference $\left(d_{-}\right.$compute $\left.t s\right)$. However, this method is vulnerable to processor sharing, which may happen on shared workers. $d_{-}$compute $t_{s}$ increases when a task yields processor to others, which leads to inaccurate measurement. We instead get $d$ _compute through measuring CPU time consumed by the engine container during the computation, written as d_cputime. 


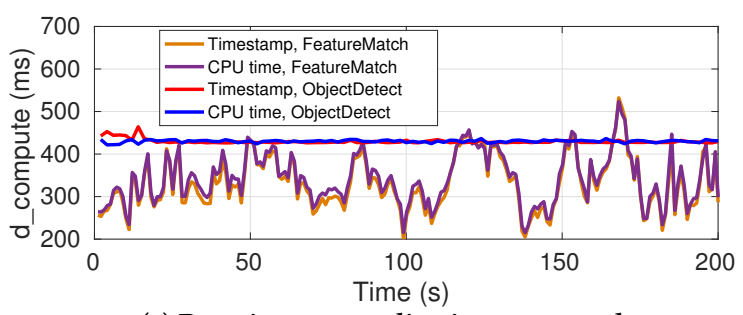

(a) Running two applications separately.

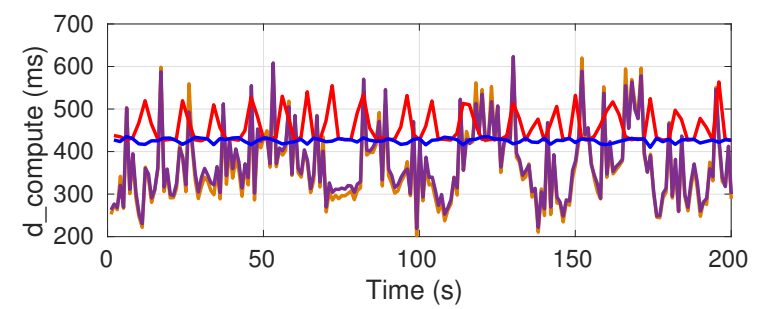

(b) Running two applications concurrently.

Figure 4: Measure computing time using timestamps (d_compute $\left.e_{t s}\right)$ and CPU time $\left(d_{-}\right.$compute $\left.e_{c p u}\right)$ for FeatureMatch and ObjectDetect. It shows that $d_{-}$compute $t_{s}$ is influenced by interference and $d_{-}$compute $e_{c p u}$ is robust.

The computing time is obtained by:

$$
d_{-} \text {compute } e_{c p u}=d \_c p u t i m e / T \_p a r a l
$$

The OS stops counting CPU time when a process turns to sleep, so only the running time of computing engine is measured. We demonstrate the robustness of $d \_c o m p u t e_{c p u}$ against the influence caused by processor sharing through an example. Figure 4a gives computing time of FeatureMatch and ObjectDetect that run separately, measured by timestamps and CPU time respectively on a 8-core machine (Intel i7-4790, 3.6GHz). We use $T$ _paral $=1.7$ for FeatureMatch and $T \_$paral $=7.3$ for ObjectDetect to fit Equation 2 to make $d_{-}$compute $e_{c u}$ accord with $d_{-}$compute ${ }_{t s}$. In Figure $4 \mathrm{~b}$ the two applications run concurrently and induce interference due to processor sharing. For ObjectDetect, $d_{-}$compute $_{t s}$ has an evident fluctuation and deviates from $d_{-}$compute $e_{p u}$, because it yields processor to FeatureMatch. The deviation is smaller for FeatureMatch because it rarely yields processor under the completely fair scheduling of Linux [24]. The result also shows that additional fluctuation caused by interference arises in $d_{-}$compute $e_{c p u}$ of FeatureMatch when the two applications runs concurrently, which again addresses the need to reduce processor contention.

Heterogeneous workers: Because tasks of a client can run on a cluster of workers, if workers have different types of processors (e.g. number of cores, clock rate), the measured d_compute is machinedependent and can not be directed used for time series prediction. Even for application with constant computing time, $d$ _compute may vary for different machines. To handle this, we use a simple approach with the assumption that a more powerful worker speeds up a task linearly. The speedup factor $s_{k}$ is a constant for worker $k$ that the following equation is satisfied for any two workers:

$$
s_{1} \cdot d \_ \text {cputime } 1=s_{2} \cdot d \_ \text {cputime } 2
$$

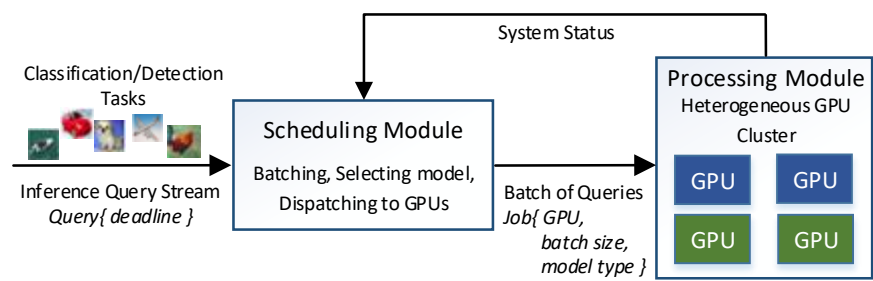

Figure 5: The architecture of DNN inference server.

The predictor uses $s \cdot d \_c p u t i m e$ as samples instead of $d \_c o m p u t e$. Then the prediction result is divided by $s_{k}$ to obtain the predicted d_cputime, and Equation 2 is used to get the computing time (denoted as d_compute').

\subsection{Serving DNNs on GPUs}

A DNN serving system deploys trained models on GPU devices. It uses dynamic batching [7, 16], as well as multi-tasking techniques on GPUs $[2,16,25,35]$, to improve resource utilization and thus processing throughput. In this work we only use batching for throughput improvement. The system is shown in Figure 5. It processes a continuous stream of incoming queries sent by clients, which are DNN inference tasks. The GPU cluster may contain different types of GPUs, where each GPU processes batches of tasks sequentially. The adaptive batching technique groups tasks together and processes them in a single DNN inference pass. The batch size is dynamically decided by the scheduler that optimizes QoS based on the timing and accuracy requirements of tasks. A task comes with soft and hard deadlines $\left(t_{d d l}^{s}\right.$ and $\left.t_{d d l}^{h}\right)$ specified by the application, which give the time budget to process the task for use in adaptive batching. The details of adaptive batching are given in Section 7 .

\section{TIMING IN THE SYSTEM}

Our framework needs accurate timing information for the PlanSchedule algorithm. In a distributed system, delays and uncertainties of network communications and clock offsets are two main sources of timing uncertainties. We use estimation techniques to infer their values to use in the scheduling algorithms.

\subsection{Estimation on Upstream Latency}

We estimate network delays $d_{u p}$ using The TCP retransmission timeout estimator [18]. We denote the value to predict as $y$. The estimator keeps a smoothed estimation $y^{s}$ and a variation $y^{\text {var }}$. They are updated by a new sample $y_{i}$ as:

$$
\begin{aligned}
y^{v a r} & \leftarrow(1-\beta) \cdot y^{v a r}+\beta \cdot\left|y^{s}-y_{i}\right|, \\
y^{s} & \leftarrow(1-\alpha) \cdot y^{s}+\alpha \cdot y_{i} .
\end{aligned}
$$

The upper bound $y^{u p}$ is given by:

$$
y^{u p}=y^{s}+\kappa \cdot y^{v a r},
$$

where $\alpha, \beta$ and $\kappa$ are parameters. In additional to delay values, because network delays $d_{u p}$ may have large fluctuations, we estimate the lower and upper bounds $\left(d \_u p^{l o w}, d \_u p^{u p}\right)$ as well. We use subtraction instead of addition in Equation 6 to obtain the lower bound. The estimator has a non-zero error when a new sample of $d_{u p}$ falls out of the bounds, calculated as its deviation from the nearest 


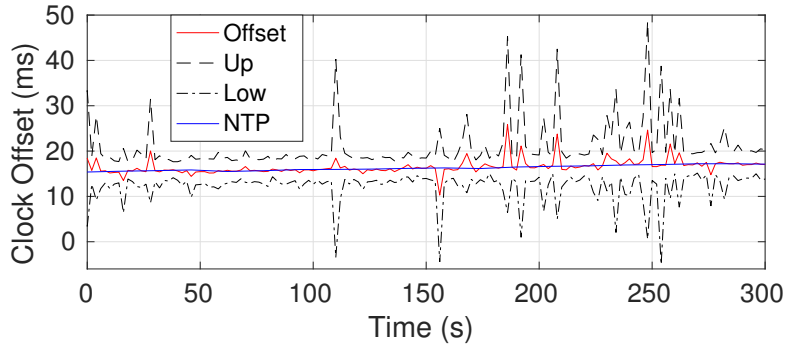

Figure 6: Clock synchronization results. NTP requests are sent every $2 s$, the offset (red) with lower and upper bounds calculated from single NTP response are given. The result of NTP algorithm using $N_{n t p}=8$ (blue) is invulnerable to RTT fluctuation.

bound. The error is positive if $d_{u p}$ exceeds $d \_u p$, and negative if it is smaller than $d \_u p^{l o w}$. Tuning the estimator parameters is a tradeoff between error and estimation uncertainty $\left(d \_u p^{u p}-d \_u p^{l o w}\right)$. Because the uncertainty is included into task reservation, larger uncertainty overclaims the reservation time slot, which causes higher interval jitters and a lower processor utilization.

\subsection{Clock Synchronization}

We adopt a general solution for clock synchronization that does not need any patch on mobile client-end devices. The ATOMS master is synchronized to the global time using NTP. Because time service now is ubiquitous on mobile devices, we require clients to be coarsely synchronized via NTP either to the global time or to the ATOMS master. We use the following approach for fine clock synchronization. Client sends out a NTP synchronization request to the master each time it receives offloading result to avoid the wake-up delay [27]. Although the client can calculate clock offset using just each single NTP response, in order to eliminate the influence of packet delay spikes, it buffers $N_{n t p}$ responses and runs the NTP algorithm [21]. It applies clock filter, selection, clustering and combining algorithms to $N_{n t p}$ responses and finally outputs a robust estimate on clock offset. ATOMS uses the estimated clock offset to synchronize timestamps exchanged between clients and the master.

In Figure 6, we demonstrate the clock synchronization on one client. The client gets an NTP response per offloading cycle (2s). The values of offset, lower and upper bounds (offset $\pm R T T / 2$ ) are plotted as the red and two dashed black lines respectively. Due to spikes in RTT of NTP packets, the offset of single response has frequent fluctuation and is thus less reliable. The blue line gives the offset obtained by the NTP algorithm using batches of $N_{\text {sample }}=8$ responses. It proves that our approach is able to get rid of RTT uncertainties and obtains a robust estimate of clock offset.

\section{PLAN-SCHEDULE ALGORITHM}

Figure 1 gives an example of the method that adjusts task start time to reduce CPU contention. A client offloads an object detection task to a server serving a group of clients. The task may be queued before running due to a shortage of computing resource. By accurately predicting the contention, the mobile client can postpone offloading

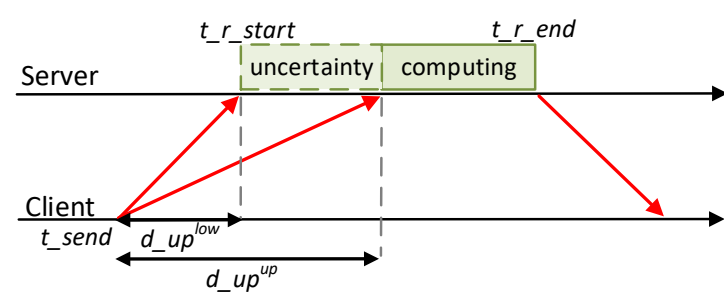

Figure 7: Processor reservation for a future offloaded task includes the uncertainty of arriving time at the server and its computing time.

and provide a more recent scene (blue box) that better localizes the moving car (green box). We describe the method, named PlanSchedule, in this section.

\subsection{Offloading Workflow}

Task adjustment in the Plan-Schedule algorithm is done through three steps: creating reservation, planning, and scheduling.

Reservation: Before the master planning a future task $T_{j}$, it creates a new reservation $R_{j}=\left(t_{-} r_{-}\right.$start ${ }_{j}, t_{-} r_{-} e n d_{j}, T_{-}$paral $\left._{j}\right)$, where $t_{-} r_{-}$start $_{j}$ and $t_{-} r_{-} e n d_{j}$ are the start and end times, and $T_{-}$paral ${ }_{j}$ is the demanded cores. Figure 7 shows the time range that a reservation covers. Given the lower and upper bounds of upstream network delay $\left(d_{-} u p_{j}^{l o w}, d_{-} u p_{j}^{u p}\right)$ estimated by the master, as well as the predicted computing time (d_compute ${ }_{j}^{\prime}$ ), the span of reservation is calculated as:

$$
\begin{gathered}
t t_{-} r_{-} \operatorname{start}_{j}=t_{-} \operatorname{sen}_{j}+d_{-} u p_{j}^{l o w}, \\
t r_{-} e n d_{j}=t_{-} s e n d_{j}+d_{-} u p_{j}^{u p}+d_{-} \text {compute }{ }_{j}^{\prime} .
\end{gathered}
$$

Reservation $R_{j}$ contains the uncertainty of the time when $T_{j}$ arrives at the server $\left(t_{-}\right.$server $\left._{j}\right)$, and the time consumed by computation. Provided that the predictions on network delays and computing times are correct, the future task will fall into the reserved time slot.

Planning: The planning step runs before offloading a future task. It coordinates future tasks of all clients to ensure that the total amount of all reservations never exceeds the limit of total processor resources of all workers. The master creates a reservation for task $T_{j}$ and plans it when:

$$
t_{\text {now }}=t_{-} r_{-} \text {start }_{j}-d \_ \text {future, }
$$

where $t_{n o w}$ is the master's current clock time, and $d \_$future is a parameter specifying how far after $t_{\text {now }}$ that the planning phase covers. The planner predicts and coordinates all future tasks starting before $t_{\text {now }}+d$ future.

$T_{\text {next }}^{i}$ is the next task of client $C_{i}$ to start the planning step. The master server plans future tasks in the ascending order of start time $t_{-} r_{\text {s start }} i_{\text {next }}$. Given that $C_{i}$ has a new task to be planned with the earliest $t_{-} r_{-} s t a r t_{\text {next }}^{i}$, the planner creates a new reservation $R_{\text {next }}^{i}$. The next task to plan for $C_{i}$ is updated to $T_{\text {next }+1}^{i}$ with a new sending time $t \_s e n d_{\text {next }+1}^{i}=t \_s e n d_{\text {next }}^{i}+d \_$period ${ }_{i} . R_{\text {next }}$ is the input of the planning algorithm. It detects resource contention, and reduces that by adjusting the sending times of both the new task and a few planned tasks. The details will be given in Section 6.2. 
A reservation $R_{j}^{i}$ is adjustable until $t_{\text {now }}=t \_$send $_{j}^{i}-d \_$inform $_{i}$, where $d_{-}$inform $_{i}$ is a parameter of $C_{i}$ for when the master should end adjusting a task and inform the client about the adjusted task sending time. The planner then removes $R_{j}$ and notifies the client. Upon receiving $t_{-}$send $d_{j}$, the client sets a timer to offload $T_{j}$. To guarantee that the client receives $t_{-}$send $d_{j}$ before this sending time, d_inform should be larger than downstream network delays. On the other hand, a large $d$ _inform causes reservations being removed and becoming unadjustable too early, which limits the performance of the planning algorithms.

Impact on Offloading Intervals: Offloading interval represents the time span between successive offloaded tasks of a client, calculated as:

$$
d_{-} \text {interval }_{j}=t \_ \text {send }_{j}-t \_ \text {send }_{j-1} \text {. }
$$

Because the planning step that adjusts task start times deviates the offloading interval from the period specified by the client, the intervals become non-constant. We desire smaller interval jitters that make the application performance stable and predictable, which is given by:

$$
d \_ \text {jitter }_{j}^{i}=d \_ \text {interval } l_{j}^{i}-d \_ \text {period }_{i} .
$$

Jitters are controlled in ATOMS by user provided Service-LevelObjectives (SLOs). The details are given in Section 6.2.

Scheduling: The client offloads $T_{j}$ to the master when the timer at $t_{-}$send $_{j}$ timeouts. After receiving the task, using the information of currently running tasks on each worker, the scheduler selects the worker that has the least processor contention. The contention $\Phi_{k}$ on worker $W_{k}$ is calculated by:

$$
\Phi_{k}=\int_{t_{\text {now }}}^{t_{-} \text {end }}{ }_{\text {new }}^{\prime} \max \left(\operatorname{load}(t)-W_{-} c p u_{k}, 0\right) \cdot d t .
$$

The master dispatches the task to the worker for processing and sends the result back to the client.

\subsection{The Planning Algorithms}

Created reservations are buffered in reservation queues, which represents a portion of processor resource in the cluster for contention detection. A queue $Q_{k}$ has a resource limit $Q_{-} c p u_{k}$ with CPU core as the unit. Each computing engine is assigned to a reservation queue. The sum of $Q_{-} c p u$ of all queues is equal to the total cores in the cluster.

Contention Detection: The planning algorithm first selects a queue to insert a new reservation $R_{\text {new }}$ in. It iterates over all queues, for $Q_{k}$, calculates the needed amount of time $\left(\Delta_{k}\right)$ to adjust $R_{n e w}$. It also calculates the total processor usage $\left(\Theta_{k}\right)$ of $Q_{k}$ during the time slot of $R_{\text {new }}$. The planner selects the queue with the minimal $\Delta$, calculated using Algorithm 1, where $\delta$ is a change of processor load $v$ at a time $t$. It checks whether the total load on $Q_{k}$ after adding $R_{\text {new }}$ exceeds the limit $Q \_c p u$. If so, the algorithm calculates $\Delta_{k}$ : the contention can be eliminated after postponing $R_{n e w}$ by $\Delta_{k}$. Otherwise $\Delta_{k}=0$.

SLOs: In the next step to adjust task start times, rather than simply postponing $R_{n e w}$ by $\Delta$, the planner moves ahead a few planned reservations as well. The coordination process takes $\Delta$ as input and adjusts reservations according to cost ( $R_{-} \operatorname{cost}$ ), a metric on how far the measured offloading interval $d$ _interval deviates from the client's SLOs, which is a list of desired percentiles of $d$ _interval. For

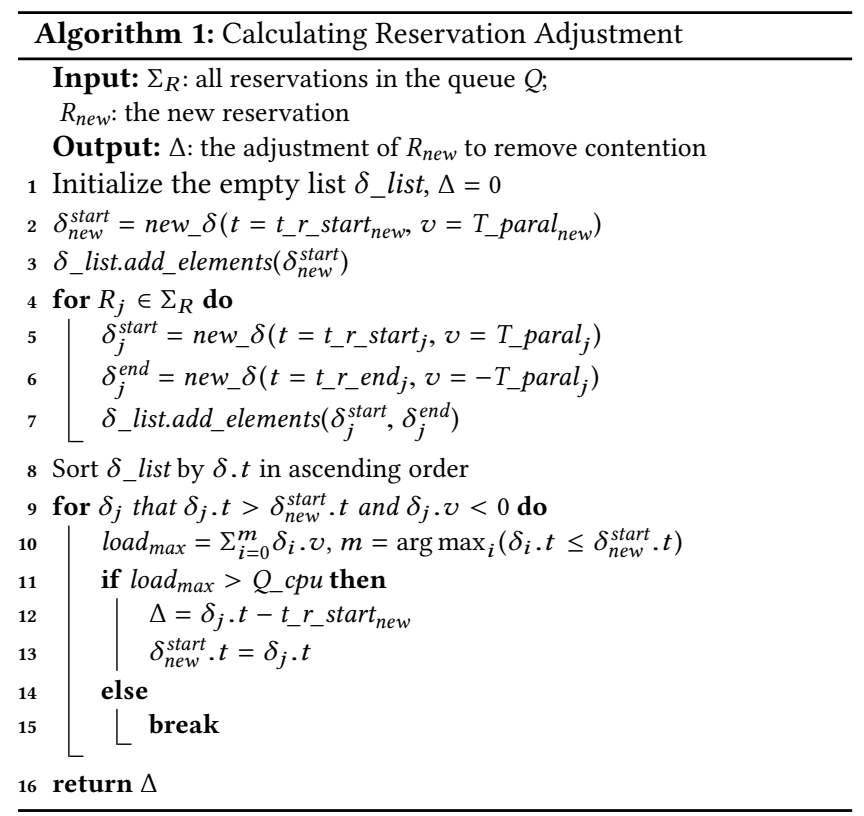

example, a client with period 1 s may require a lower bound $d \_s l 0^{10 \%}$ $>0.9 \mathrm{~s}$ and an upper bound $d \_s l^{90 \%}<1.1 \mathrm{~s}$. The master calculates $R \_$cost using the measured percentiles of interval (d_interval $\left.l^{p}\right)$. For the new reservation $\left(R_{\text {new }}\right)$ to be postponed, the cost $R_{-}$cost $t^{+}$is obtained from the upper bounds:

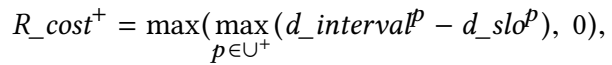

where $p$ is a percentile and $\mathrm{U}^{+}$is the set of percentiles that have upper bounds in the SLOs. $R \_$cost ${ }^{+}$is the maximal interval deviation from the SLOs. For tasks to be moved ahead, the deviation from lower bounds $\left(\mathrm{U}^{-}\right)$are used instead to obtain the cost $R \_$cost $t^{-}$.

Reservation Coordination: reservation block $(B)$ is the basic unit of coordination. It simplifies the process comparing with adjusting reservations individually. The slack between two reservations $R_{i}, R_{j}$ where $i<j$ is slack $_{i, j}=t \_r \_s t a r t_{j}-t_{-} r_{-} e n d_{i}$. Any two reservations with no slack in between are merged into the same block. The span of a block from $t \_b \_s t a r t$ to $t \_b \_e n d$ covers all reservations in it. Algorithm 2 describes the process to adjust tasks. It starts with the last two blocks, and iteratively adjusts two adjacent blocks in reverse order. The amount of time to move away two blocks, initialized to $\Delta$ given by Algorithm 1, is divided proportionally according to $B$ _cost. It is equally divided if both costs are zero. Moving a block ahead reduces the slack between it and the previous block. Two blocks are merged when the slack becomes zero. The process ends if a block has enough slack to move ahead or it is already the first block in queue. After obtaining the adjustment of reservation $R_{j}$, the change is applied to the sending time ( $t \_s e n d_{j}$ ) of the associate task that will be sent to the client.

\subsection{Predicting Computing Times}

Accurate prediction of computing time is essential for resource reservation. We use upper bound estimation for applications with 


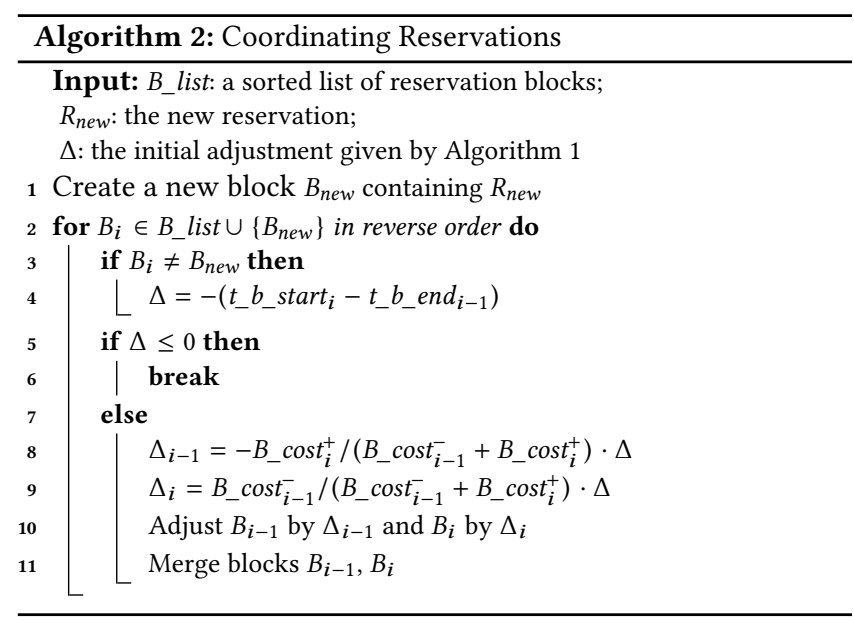

low variability of computing time, and time series prediction for applications with high variability. Given that $T_{n}$ is the last completed task of client $C_{i}$, instead of just predicting $T_{n+1}$ (the next task to run), ATOMS needs to predict $T_{\text {next }}$ (the next task to plan, next $>n$ ). $N_{\text {predict }}=n e x t-n$ gives how many values it needs to predict since the last sample. It is decided by the parameter $d \_$future (Section 6.1) and the period of the client, calculated as $\left\lceil d \_\right.$future/d_period $\left.d_{i}\right\rceil$.

Upper Bound Estimation: The first method estimates the upper bound of samples using a TCP retransmission timeout estimation algorithm [18], as we have described in Section 5.1. This lightweight method is adequate for applications with low computing time variability, such as ObjectDetect. It tends to overestimate for applications with highly varying computing time as it uses upper bound as the prediction.

Time Series Linear Regression: In the autoregressive model for time series prediction problems, the value $y_{n}$ at index $n$ is assumed to be a weighted sum of previous samples in a moving window with size $k$. That is,

$$
y_{n}=b+w_{1} y_{n-1}+\cdots+w_{k} y_{n-k}+\epsilon_{n},
$$

where $y_{n-i}$ is the $i$ th sample before the $n$ th, $w_{i}$ is the corresponding coefficient and $\epsilon_{n}$ is the noise term. We use this model to predict $y_{n}$. The inputs $\left(y_{n-1}\right.$ to $\left.y_{n-k}\right)$ are the previous $k$ samples measured by workers. We use a recursive approach to predict the $N_{\text {predict }}$ th sample after $y_{n-1}$ : to predict $y_{i+1}$, the predicted $y_{i}$ is used as the last sample. This approach is flexible to predict arbitrary future samples, however, as $N_{\text {predict }}$ increases, the accuracy degrades because the prediction error is accumulated.

The predictor keeps a model for each client which is trained either online or offline. In the online approach, previous samples are collected as mini-batches of training data. The model is retrained on the latest mini-batches. The offline approach is simpler which trains the model once on a pre-collected series of samples. We predict the computing time for FaceDetect as an example. The video input is from Jiku dataset [29] with a frames per second (FPS) equal to 2 . The cumulative distribution functions (CDFs) of prediction errors (d_compute $\left.{ }^{\prime}-d \_c o m p u t e\right)$ for $N_{\text {predict }}=1,3,5$ are given in Figure 8. It shows that compared with linear regression, upper bound estimation tends to overestimate when $N_{\text {predict }}=1$.
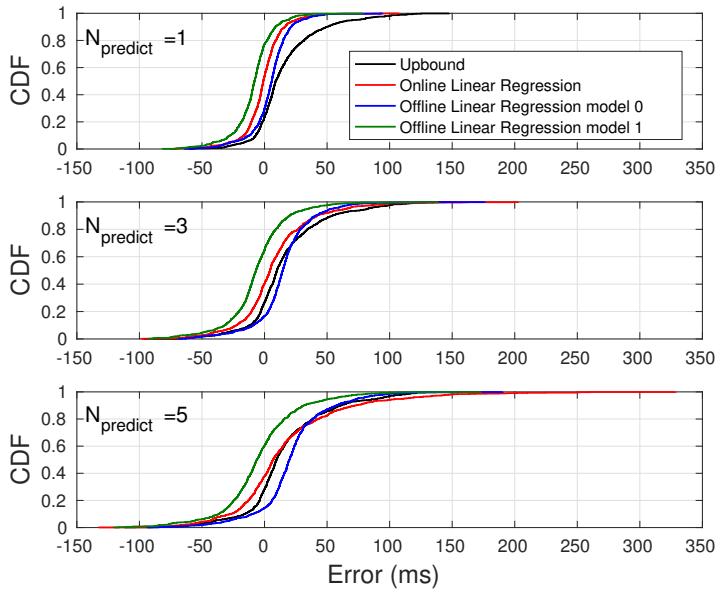

Figure 8: CDFs of prediction errors.

However, the performance of linear regression degrades for larger $N_{\text {predict }}$. We also give the errors using two offline models trained on videos with different context in Figure 8. Compared with the online method, offline model 0 tends to overestimate and offline model 1 tends to underestimate. This suggests that the performance of offline model is sensitive to the content of the video which generates the training data.

\subsection{Evaluating ATOMS}

We conduct several experiments to evaluate ATOMS. It is compared with a First-Come-First-Serve (FCFS) system that processes tasks without using the planning algorithms.

Micro Testbed: We first evaluate the use of ATOMS on a micro testbed serving the ObjectDetect application. It serves 4 RaspberryPi boards as clients $\left(C_{0,1,2,3}\right)$, with offloading periods $2 \mathrm{~s}, 3 \mathrm{~s}, 4 \mathrm{~s}$ and $5 \mathrm{~s}$. They connect to the ATOMS master, which runs on a 4-core (Intel i5-6600, 3.3GHz) desktop through public campus Wi-Fi hotspot. There is one worker machine (8-core, Intel i7-4790, 3.6GHz) hosting a computing engine of ObjectDetect. Clients start at random initial times. The planning length of ATOMS is $d \_$future $=6 \mathrm{~s}$. The inputs of ObjectDetect are from multi-camera pedestrians videos [13]. In Figure 9, the delay plot (bottom) shows that the multitenant interference aggravates E2E delay of all the clients. ATOMS effectively mitigates the interference and significantly improves the delay performance. On the other hand, offloading intervals become non-constant, caused by task adjustments.

Longer delays cause trackability loss in object detection. To demonstrate this, we use intersection over union (IOU) [9] as the metric of successful detection. The precision is the ratio of accurately detected objects $(I O U>50 \%)$ using offloading over detected objects by the scheme itself [6]. The video inputs of four clients are taken by cameras under different angles filming the same crowd of pedestrians. Trackability varies for different videos due to the difference of pedestrian moving speeds. The result in Figure 10 shows that ATOMS improves precision for all clients, where $C_{0}$ gets the largest precision improvement (over 20\%), because it suffers from the worst delay without ATOMS. 

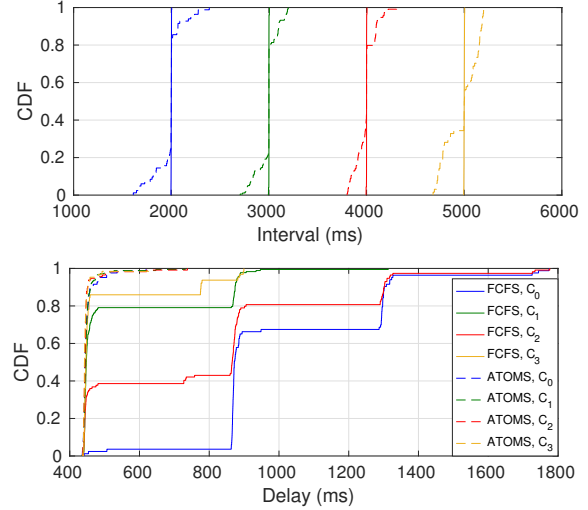

Figure 9: Effects of ATOMS in offloading interval (top) and E2E delay (bottom). The average CPU utilization is $50 \%$.

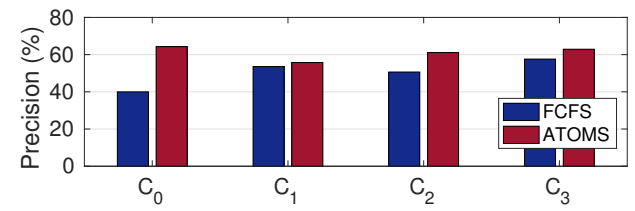

Figure 10: Precision of pedestrian detecting and tracking.

Scaling-Out: We use ATOMS to serve more clients on the AWS testbed. The master runs on a c4.xlarge instance (4 vCPUs). Each worker machine is a c4.2xlarge instance ( 8 vCPUs). There are 12 to 24 clients running FaceDetect with periods from $0.5 \mathrm{~s}$ to $1.0 \mathrm{~s}$, under Wi-Fi networks. $d$ future $=2 \mathrm{~s}$ is used in planning. We use one worker machine ( 8 vCPUs) hosting 8 FaceDetect engines. The planner has a reservation queue for each engine with $Q_{-} c p u=1$. As shown in Figure 11, the interference becomes more intensive as the number of clients becomes larger, and FCFS suffers from increasingly longer E2E delay. ATOMS is able to maintain low E2E delay even when the total CPU utilization is over $80 \%$. Using the case with 24 clients as example, the $90 \%$ percentile of E2E delay is reduced by $34 \%$ in average for all clients, and the maximum reduction is $49 \%$. The interval plots (top) show that offloading interval jitter increases in ATOMS which is caused by task adjustments.

For more results and discussions on using the Plan-Schedule algorithms for a heterogeneous cluster of worker machines, we refer readers to our works in [11].

\section{ADAPTIVE BATCHING OF DNN INFERENCE}

For DNN workloads on GPUs, the system packs offloaded tasks using the same DNN model as a batch and selects the suitable model to run on GPUs. The batch size and DNN model type are selected to optimize QoS considering task accuracy and delay. We consider two QoS-aware schedulers: a heuristic scheduler and a more advanced one using deep reinforcement learning.

\subsection{Improving Throughput using Batching}

We first discuss the impact of batching on processing throughput of DNNs through measurements. Two models are used: the full model achieves a mean average precision (mAP) of $63.4 \%$ on the
PASCAL VOC dataset [10] and the mAP is $57.1 \%$ for the less complex tiny model [3]. We measure $D_{B, M, G}$ on AWS g2.2xlarge (g2) and more powerful p2.xlarge (p2) GPU instances. ${ }^{1}$ The measured throughputs in queries per second (QPS) are given in Table 1, with $B$ ranging from 1 to $64, M \in\{$ tiny, full $\}$, and $G \in\{\mathrm{g} 2, \mathrm{p} 2\}$. It shows that batching effectively improves the throughputs.

Table 1: DNN Inference Throughputs (QPS)

\begin{tabular}{c|c|ccccccc}
\hline $\mathrm{G}$ & $\mathrm{M}$ & $\mathrm{B}=1$ & 2 & 4 & 8 & 16 & 32 & 64 \\
\hline \multirow{2}{*}{$\mathrm{g} 2$} & tiny & 25.6 & 35.7 & 51.9 & 66.1 & 73.7 & 84.7 & 91.2 \\
& full & 9.4 & 9.4 & 12.3 & 14.7 & 16.0 & 16.8 & - \\
\hline \multirow{2}{*}{ p2 } & tiny & 23.8 & 35.7 & 63.5 & 90.9 & 109.6 & 131.1 & 141.9 \\
& full & 14.5 & 18.9 & 24.2 & 28.4 & 31.5 & 33.9 & 34.6 \\
\hline
\end{tabular}

\subsection{QoS Metrics}

The scheduler dynamically selects a batch size and a DNN model to process on a GPU in a cluster. Its goal is to maximize the realtime system performance that is a utility function of inference accuracy, delay, and task deadline. The quality of processing a task $(Q)$, defined as a function of the task completion time $\left(t_{\text {end }}\right)$, the soft and hard deadlines $\left(t_{d d l}^{s, h}\right)$, and the DNN model $(M)$, is computed as:

$$
Q\left(t_{\text {end }}, t_{d d l}^{s, h}, M\right)=\kappa_{M} \cdot V\left(t_{\text {end }}, t_{d d l}^{s, h}\right),
$$

where $\kappa_{M} \in(0,1]$ is a factor to penalize less accurate models that we call accuracy weight. $V$ is a time-utility function (TUF) to represent the influence of delay on response quality. The function $V$ that we use is illustrated in Figure 12: the utility value of a response is 1 if $t_{\text {end }}$ meets the soft deadline, otherwise it decreases linearly until zero after passing the hard deadline.

\subsection{QoS-Aware Adaptive Batching}

The scheduler prioritizes tasks with earlier deadlines to avoid missing deadlines. To efficiently order tasks according to deadlines on a heavily loaded server, we use an approximate sorting method. We quantize time into discrete bins, as shown in Figure 13. The duration of each bin is $\Delta t$. A task with soft deadline $t_{d d l}^{s}$ is allocated to bin $i$ with $i=\left(t_{d d l}^{s}-t_{\text {init }}\right) / \Delta t$, where $t_{\text {init }}$ is the system initialization time. The bins are created and deleted dynamically. The scheduler fetches tasks starting from the bin with the smallest indexes (earliest deadlines).

Heuristic Method: The heuristic scheduler always selects the smallest batch size $(B)$ that satisfies the requirement on throughput:

$$
B \geq\left[\alpha \cdot\left(D_{B, M, G} \cdot R_{t}\right)+(1-\alpha) \cdot N_{t}\right] \cdot \beta \cdot p_{G},
$$

where $R_{t}$ is the arriving rate of tasks at time $t$. The scheduler allocates tasks to GPUs proportionally to their processing capabilities. $p_{G} \in(0,1]$ is the portion of tasks allocated to GPU $G$. We use the processing throughput of $B=8$ to measure the capability of a GPU to obtain $p_{G}$. The term $D_{B, M, G} \cdot R_{t}$ represents the number of incoming tasks. $N_{t}$ is the total number of buffered tasks. $\alpha \in[0,1]$ and $\beta>0$ are tuning parameters tuned on training data.

The scheduler uses the most accurate DNN model by default. If the batch size is not less than a threshold $B_{t h r d}=8$, the scheduler

${ }^{1}$ GPU type is NVIDIA GRID for $\mathrm{g} 2.2 \mathrm{xlarge}$ and NVIDIA GK210 for p2.xlarge. 

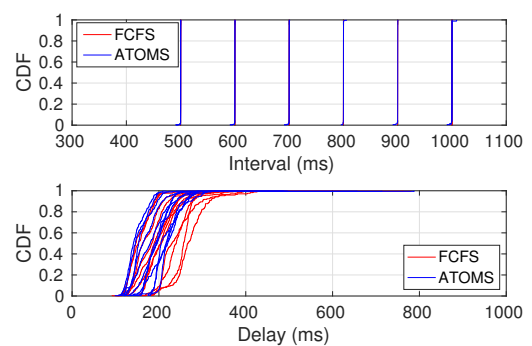

(a) 12 clients. The mean CPU utilization is $38 \%$ for FCFS and $36 \%$ for ATOMS.

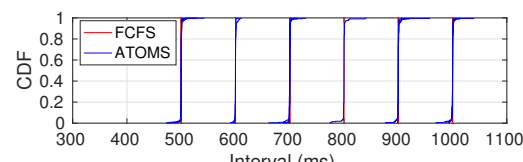

Interval (ms)

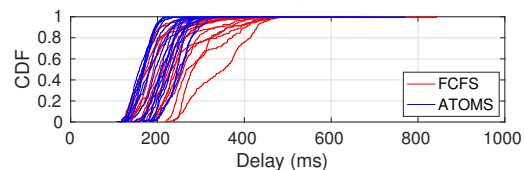

(b) 18 clients. The mean CPU utilization is $58 \%$ for FCFS and 55\% for ATOMS.
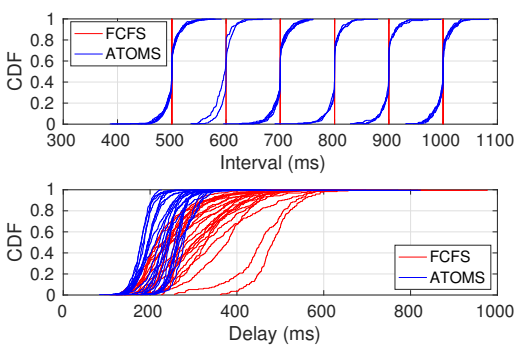

(c) 24 clients. The mean CPU utilization is $83 \%$ for FCFS and $81 \%$ for ATOMS.

Figure 11: Offloading performance (CDF of each client) of FCFS and ATOMS running FaceDetect using Wi-Fi.

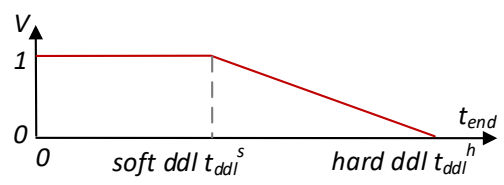

Figure 12: Utility of task response as a function of time delay $V\left(t_{\text {end }}, t_{d d l}^{s, h}\right)$.

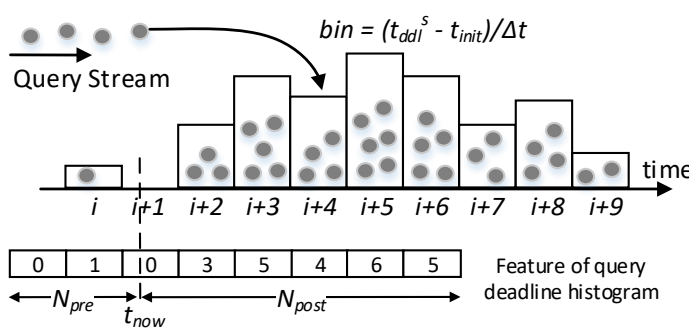

Figure 13: Buffering incoming tasks (queries) in bins to approximately order them according to deadline $t_{d d l}^{s}$.

checks all simplified model types and selects the model maximizing the total quality value of this batch:

$$
Q_{B}=\kappa_{M} \cdot \sum_{q \in B} Q\left(t_{\text {now }}+D_{B, M, G}, t_{d d l}^{s, h}, M\right) .
$$

Reinforcement Learning Method: In reinforcement learning, the agent observes the state $s_{t}$, chooses the action $a_{t}$, and obtains a reward $r_{t}$ gained from this action, at time $t$. The learning process aims at maximizing the expected cumulative reward: $\mathbb{E}\left[\sum_{t=0}^{\infty} \gamma^{t} r_{t}\right]$, where $\gamma \in(0,1]$ is the discount factor of future reward. The agent selects actions based on a policy $\pi(s, a)$ that gives the probability to perform $a$ in state $s$. A deep RL agent uses a DNN to approximate the policy $\pi(s, a)$. Here we describe state, action, and reward to customize this deep RL agent in our scheduling problem:

State represents the status of the system. It includes the following four parts:

Histogram of task deadlines: We use a histogram of task deadlines as the feature representing urgency of buffered tasks. The feature vector is obtained from the sizes of task bins. As shown in Figure 13, at the current time $t$ that corresponds to the bin $i$, the histogram feature comprises of $N_{\text {pre }}$ bins before and $N_{\text {post }}$ bins after the bin $i$. The size is zero for a non-existent bin, hence this feature has a fixed length $N_{\text {pre }}+N_{\text {post }}$. Because the histogram state has been truncated to a fixed length, it may be less than the real total number of buffered tasks. Therefore we add the total number of buffered tasks as an additional feature.

Arriving rate: The state contains the arrival rate $\left(R_{t}\right)$ of tasks from all clients to estimate the number of incoming tasks in future.

GPU type: The scheduler must consider the processing capability of the GPU that processes the batch. We use a binary vector to represent the GPU type: an element $e$ of the vector is 1 if the GPU matches the type that $e$ is associated with, otherwise $e$ is 0 .

Availability of GPUs: When dispatching a batch to a GPU, the optimal batch size is influenced by the availability of other GPUs. We represent availability as the expected rest computing time of each device $\left(t_{\text {end }}-t_{\text {now }}\right)$, where $t_{\text {end }}$ is estimated using the measured mean computing time (see Table 1). The state includes the availabilities of all GPUs.

An action selects a batch size $B$ and a model type $M$, then runs the batch on a GPU. Reward is defined as the average quality (Equation 15) of the latest $W$ tasks. We follow the routine of a policy gradient RL algorithm to train the model [32]. In our implementation, the policy model of the RL scheduler has 1 fully connected hidden layer with 64 neurons. The histogram of deadlines feature in the state uses $N_{\text {pre }}=4$ and $N_{\text {post }}=6$. The reward is measured using the qualities of last $W=1000$ tasks.

\subsection{Evaluating Adaptive Batching}

In the evaluations, we emulate the DNN inference server on a simulation platform. A task runs either the full or the tiny YOLO object detection model. We use accuracy weights $\kappa_{\text {full }}=1$ and $\kappa_{\text {tiny }}=0.5$. The task stream is generated from a web query trace. ${ }^{2}$ The evaluations are done by using queries from 4 logs from 12 hours in one day (12hr to $24 \mathrm{hr}$ ), each lasts for $3 \mathrm{hr}$. Deadlines $t_{d d l}^{s}=1 \mathrm{~s}$ and $t_{d d l}^{h}=2 \mathrm{~s}$ are applied to all tasks.

We consider a baseline scheduler (denoted as fixed) for performance comparison. It batches all tasks to run on a GPU, with 32 as the maximal batch size, and selects the model that optimizes QoS. Single GPU: We first evaluate the schedulers using a processing module with one GPU (p2). Both schedulers use batch sizes as given in Table 1, and the RL scheduler has $B=0$ as an additional option.

\footnotetext{
${ }^{2}$ SogouQ: http://www.sogou.com/labs/resource/q.php.
} 
Table 2: Mean (SD) Response Quality of Single GPU

\begin{tabular}{c|c|c|c|c}
\hline Scheduler & $12-15 \mathrm{hr}$ & $15-18 \mathrm{hr}$ & $18-21 \mathrm{hr}$ & $21-24 \mathrm{hr}$ \\
\hline Fixed & $0.883(0.204)$ & $0.818(0.235)$ & $0.860(0.218)$ & $0.863(0.218)$ \\
Heuristic & $0.968(0.117)$ & $0.915(0.185)$ & $0.950(0.146)$ & $0.939(0.161)$ \\
RL & $0.978(0.084)$ & $0.931(0.149)$ & $0.961(0.114)$ & $0.949(0.132)$ \\
\hline
\end{tabular}

Table 3: $P_{\text {tiny }}(\%)$ of Single GPU

\begin{tabular}{c|c|c|c|c}
\hline Scheduler & $12-15 \mathrm{hr}$ & $15-18 \mathrm{hr}$ & $18-21 \mathrm{hr}$ & $21-24 \mathrm{hr}$ \\
\hline Fixed & 20.2 & 33.8 & 25.0 & 25.4 \\
Heuristic & 5.5 & 16.3 & 9.2 & 11.7 \\
RL & 2.0 & 8.2 & 4.1 & 6.1 \\
\hline
\end{tabular}

Table 4: Mean (SD) Response Quality of Multi-GPUs

\begin{tabular}{c|c|c|c|c}
\hline Scheduler & $12-15 \mathrm{hr}$ & $15-18 \mathrm{hr}$ & $18-21 \mathrm{hr}$ & $21-24 \mathrm{hr}$ \\
\hline Fixed & $0.820(0.231)$ & $0.767(0.242)$ & $0.801(0.237)$ & $0.813(0.234)$ \\
Heuristic & $0.948(0.148)$ & $0.883(0.210)$ & $0.925(0.176)$ & $0.916(0.186)$ \\
RL & $0.955(0.139)$ & $0.895(0.202)$ & $0.933(0.168)$ & $0.922(0.179)$ \\
\hline
\end{tabular}

Table 5: $P_{\text {tiny }}(\%)$ of Multi-GPUs

\begin{tabular}{c|c|c|c|c}
\hline Scheduler & $12-15 \mathrm{hr}$ & $15-18 \mathrm{hr}$ & $18-21 \mathrm{hr}$ & $21-24 \mathrm{hr}$ \\
\hline Fixed & 32.6 & 43.5 & 36.5 & 34.4 \\
Heuristic & 9.6 & 22.8 & 14.4 & 16.4 \\
RL & 8.2 & 20.6 & 12.8 & 14.8 \\
\hline
\end{tabular}

The bin size of task buffer is $\Delta t=200 \mathrm{~ms}$. The arrival rate of tasks $\left(R_{t}\right)$ is measured in a sliding window of $5 \mathrm{~s}$. The heuristic schedule use the parameters $\alpha=0.5$ and $\kappa=0.5$ that give the highest mean response quality on the training data.

The scheduling performance is measured with two metrics: (1) mean response quality (Equation 15) over 3 hours, given in Table 2 with standard deviation (SD); (2) $P_{\text {tiny }}$, the percentage of tasks that use the tiny model and have lower inference accuracy, given in Table 3. According to the results, the fixed scheduler that does not adapt batch size has the lowest response quality. In comparison with that, the heuristic scheduler effectively improves the mean quality. The RL scheduler achieves the best performance of all.

Heterogeneous GPU Cluster: We use a cluster of 5 g2 GPUs and 5 p2 GPUs to evaluate the performance of scheduling more servers. The task arrival rate is scaled up by 8 times in generating tasks to fill up the processing capability of the cluster. Observed from the results given in Table 4 and 5, similar to the single GPU case, the RL scheduler delivers higher response quality by using the tiny YOLO model less frequently.

\section{CONCLUSIONS}

This paper studies new scheduling techniques for real-time mobile offloading systems deployed in the cloudlet. We design and implement the ATOMS system that serves computer vision algorithms running on CPUs as micro-services. It uses the Plan-Scheduling algorithm to mitigate multi-tenant resource contention and to improve delay for CPU workloads. DNN inference tasks running on GPUs are studied as well. Adaptive batching algorithm is used to achieve the best QoS for offloaded tasks, which selects the batch size based on information of delay, model complexity and system load. The effectiveness of our systems in improving multi-tenant serving performance is demonstrated by experiments.

\section{REFERENCES}

[1] Docker. https://www.docker.com/. [Online; accessed 16-July-2018].

[2] NVIDIA Multi-Process Service. https://docs.nvidia.com/deploy/pdf/CUDA_ Multi_Process_Service_Overview.pdf. [Online; accessed 16-July-2018].

[3] YOLO Models. https://pjreddie.com/darknet/yolo/. [Online; accessed 16-July2018].

[4] H. Bay, A. Ess, T. Tuytelaars, and L. Van Gool. Speeded-up robust features (SURF). Computer Vision and Image Understanding, 110(3):346-359, June 2008.

[5] G. Bradski. The Opencv library. Dr. Dobb's Journal of Software Tools, 2000.

[6] T. Y.-H. Chen, L. Ravindranath, S. Deng, P. Bahl, and H. Balakrishnan. Glimpse: Continuous, real-time object recognition on mobile devices. In SenSys, 2015.

[7] D. Crankshaw, X. Wang, G. Zhou, M. J. Franklin, J. E. Gonzalez, and I. Stoica. Clipper: A low-latency online prediction serving system. In NSDI, 2017.

[8] E. Cuervo et al. MAUI: Making smartphones last longer with code offload. In MobiSys, 2010.

[9] M. Dantone, L. Bossard, T. Quack, and L. van Gool. Augmented Faces. In 2011 IEEE International Conference on Computer Vision Workshop (ICCVW), 2011.

[10] M. Everingham et al. The Pascal visual object classes (VOC) challenge. International journal of computer vision, 88(2):303-338, 2010.

[11] Z. Fang et al. Mitigating multi-tenant interference on mobile offloading servers: Poster abstract. In SoCC, 2017.

[12] Z. Fang et al. Qos-aware scheduling of heterogeneous servers for inference in deep neural networks. In CIKM, pages 2067-2070, 2017.

[13] F. Fleuret, J. Berclaz, R. Lengagne, and P. Fua. Multicamera people tracking with a probabilistic occupancy map. IEEE Transactions on Pattern Analysis and Machine Intelligence, 30(2):267-282, Feb 2008.

[14] K. Ha, Z. Chen, W. Hu, W. Richter, P. Pillai, and M. Satyanarayanan. Towards wearable cognitive assistance. In MobiSys, 2014

[15] S. Han et al. MCDNN: An approximation-based execution framework for deep stream processing under resource constraints. In MobiSys, 2016.

[16] J. Hauswald et al. Djinn and tonic: Dnn as a service and its implications for future warehouse scale computers. In ISCA, pages 27-40, 2015.

[17] J. Hauswald et al. Sirius: An Open End-to-End Voice and Vision Personal Assistant and Its Implications for Future Warehouse Scale Computers. In ASPLOS, pages 223-238, 2015

[18] V. Jacobson. Congestion avoidance and control. In SIGCOMM, pages 314-329, 1988.

[19] Y. Kang et al. Neurosurgeon: Collaborative intelligence between the cloud and mobile edge. In ASPLOS, pages 615-629, 2017.

[20] D. G. Lowe. Distinctive image features from scale-invariant keypoints. International fournal of Computer Vision, 60(2):91-110, Nov. 2004.

[21] D. Mills, J. Martin, J. Burbank, and W. Kasch. Network Time Protocol Version 4: Protocol and Algorithms Specification. RFC 5905 (Proposed Standard), June 2010.

[22] A. N. Modi et al. TFX: A tensorflow-based production-scale machine learning platform. In $K D D, 2017$.

[23] R. Newton, S. Toledo, L. Girod, H. Balakrishnan, and S. Madden. Wishbone: Profile-based partitioning for sensornet applications. In NSDI, 2009.

[24] C. S. Pabla. Completely fair scheduler. Linux fournal, 2009(184), Aug. 2009.

[25] J. J. K. Park, Y. Park, and S. Mahlke. Dynamic resource management for efficient utilization of multitasking gpus. In ASPLOS, pages 527-540, 2017.

[26] J. Rasley, K. Karanasos, S. Kandula, R. Fonseca, M. Vojnovic, and S. Rao. Efficient queue management for cluster scheduling. In EuroSys, 2016.

[27] L. Ravindranath et al. Timecard: Controlling user-perceived delays in serverbased mobile applications. In SOSP, 2013.

[28] J. Redmon et al. You only look once: Unified, real-time object detection. In CVPR, June 2016.

[29] M. Saini, S. P. Venkatagiri, W. T. Ooi, and M. C. Chan. The Jiku mobile video dataset. In MMSys, 2013.

[30] M. Satyanarayanan et al. The case for VM-based cloudlets in mobile computing. IEEE Pervasive Computing, 8(4):14-23, Oct 2009.

[31] C. Shi, K. Habak, P. Pandurangan, M. Ammar, M. Naik, and E. Zegura. COSMOS: Computation offloading as a service for mobile devices. In MobiHoc, 2014.

[32] R. S. Sutton, D. McAllester, S. Singh, and Y. Mansour. Policy gradient methods for reinforcement learning with function approximation. In NIPS, 1999.

[33] A. Tumanov, T. Zhu, J. W. Park, M. A. Kozuch, M. Harchol-Balter, and G. R. Ganger. Tetrisched: Global rescheduling with adaptive plan-ahead in dynamic heterogeneous clusters. In EuroSys, 2016.

[34] P. Viola and M. Jones. Rapid object detection using a boosted cascade of simple features. In CVPR, 2001.

[35] Z. Wang et al. Simultaneous multikernel gpu: Multi-tasking throughput processors via fine-grained sharing. In HPCA, pages 358-369, 2016.

[36] T. Zhang et al. The design and implementation of a wireless video surveillance system. In MobiCom, pages 426-438, 2015. 